\title{
EVALUATING A REMOTE HEALTH MONITORING APPLICATION POWERED BY BLUETOOTH
}

\author{
Maryam Vahabi, Hossein Fotouhi, Mats Björkman and Maria Lindén \\ Mälardalen University; School of Innovation, Design and Technology, Sweden
}

\begin{abstract}
It has become widely accepted that the Internet of Things (IoT) devices and technologies are the key enablers for many emerging applications including remote health monitoring. Various physiological sensing devices have been designed and equipped with different radio technologies. The choice of radio hardware plays an important role on the overall performance of the system since it imposes some limitations on the delivered quality of service. Hence, it is critical to properly evaluate the embedded radio technology based on the application requirements. In this paper, we perform extensive experiments on Shimmer physiological sensors that is one of the leading providers of wearable wireless sensor products powered by Bluetooth classic radio. Shimmer sensors are designed and used for monitoring various human health information such as temperature, heart rate, movement, etc. We review and investigate different scenarios in which Shimmer devices are used by medical practitioners to monitor the ECG signal and the movement of a human. This study shows that the Shimmer device can provide reliable data delivery by using a specific configuration. For instance, employing a maximum number of seven Shimmer devices attached on a body at home environment within the range of at most $5 \mathrm{~m}$ and with the sampling rate of $512 \mathrm{~Hz}$ would result in a reasonable quality of service, while varying these parameters may degrade the overall performance. Mobility of human body, noisy environment, and higher packet transmission rates are some examples that will reduce the system quality.
\end{abstract}

\section{KEYWORDS}

Remote Health Monitoring, Internet of Things, Wireless Communication, Bluetooth, Interference

\section{INTRODUCTION}

The advancement in the Internet of Things (IoT), wireless communication and hardware miniaturization is playing a key role in the development of remote health monitoring applications, making them more available and affordable [Doukas \& Maglogiannis, 2012]. The number of elderly people (over 60 years of age) is expected to grow more than double, from 841 million individuals in 2013 to more than 2 billion in 2050 [United Nations, 2013]. This means that the health sector will require more support from digitalization through remote health monitoring in order to reduce the cost and increase health services to elderly people. Currently, there are a lot of effort in all over the world to provide digital healthcare. For instance, in Sweden, the vision for eHealth is that in 2025, Sweden will be best in the world at using the opportunities offered by digitization and eHealth to make it easier for people to achieve good and equal health and welfare, and to develop and strengthen their own resources for increased independence and participation in the life of society [Ministry of Health and Social Affairs, 2016].

IoT is a growing community that initially was focusing only on connectivity issues, where the main idea was to connect all devices (things) to the Internet [Reiter, 2014]. Thus, IoT was an extension to the traditional wireless sensor networks (WSNs), which supports remote access to sensing devices. Though, the new IoT term covers wider area that integrates more aspects of physical devices rather than mere connectivity aspect, such as hardware design, computing power, software properties, communication protocols, data collection, data storage, data processing, data management and control loop. This way, IoT has become a multidisciplinary research area where both academia and industry are putting a lot of attention [Ray, 2018]. However, still in many IoT applications, such as remote health monitoring, one of the major research areas is providing reliable data communication over wireless medium with high QoS demands, while tackling various 
challenges, such as limited resources in terms of channel bandwidth, processing, battery power and memory [Premsankar et al., 2018], [Gardašević et al., 2017], [Fotouhi et al., 2016].

Due to the growing number of IoT devices, it is highly desired to devise algorithms and protocols in order to cope with channel bandwidth limitation, especially when devices are sharing same frequency band [Chen \& Kunz, 2016]. Most of indoor-used IoT devices operate at $2.4 \mathrm{GHz}$ frequency, which is shared among various wireless technologies, such as IEEE 802.15.4, Bluetooth, WiFi, etc. [Natarajan et al., 2016]. There exist various technologies that is used for wearable wireless network as in remote health care applications such as IEEE 802.11a/b/g/n (WiFi), IEEE 802.15.1 (Bluetooth), IEEE 802.15.1 (Bluetooth-LE), IEEE 802.15.4 (Zigbee), IEEE 802.15.4a (UWB), IEEE 802.15.4j (MBAN), IEEE 802.15.4j (MBAN) [Alam et al., 2016]. IoT devices with low-power transmission (e.g. 802.15.4 and Bluetooth) are more prone to packet losses when operating in the vicinity of higher power transmission devices such as WiFi and microwave. Thus, it is necessary to set up strategies to achieve better performance while dealing with the interference and noisy environment.

Bluetooth is one of the technologies that is widely used in medical devices. As stated earlier, it operates in 2.4 GHz frequency band and supports short range of communication (10 to $100 \mathrm{~m}$ ) with $1 \mathrm{Mbps}$ data rate for a network of at most 8 nodes [Baker, 2005]. No sleep cycle is implemented, which permits data streaming through different sensors, such as Electromyogram (EMG), Electroencephalogram (EEG) and Electrocardiogram (ECG). These features make Bluetooth a suitable choice for remote health monitoring services that requires data streaming. Shimmer device is an example of a physiological sensing device equipped with classical Bluetooth radio, which is widely used in research and development [Gardašević et al., 2017], [Ahmed, 2017], [Santos, 2016], [Sarkar \& Misra, 2016]. It can monitor the status of various health elements by measuring physiological parameters such as ECG, EMG, Electrodermal Activity (EDA), heart rate and Galvanic Skin Response [Tomasic et al., 2017], [Shimmer, 2019].

Contributions. This paper focuses on evaluating the Bluetooth classic radio of Shimmer sensor devices in various network and environment setup. Main parameters for the evaluation are number of nodes, sampling rate, mobility of nodes, and different sources of interference including the presence of human body. The results of experiments are shown in terms of packet reception rate (PRR) where together with the received signal strength indicator (RSSI), more insight into the experimental environment can be obtained.

Organization of the paper. Section 2 describes the system architecture, followed by the results and discussions of various experimental scenarios in Section 3. Finally, Section 4 concludes the paper.

\section{SYSTEM ARCHITECTURE}

This section explains the system architecture of the considered remote health monitoring ecosystem. Figure 1 depicts a schematic view of all the components used in our system design. In such systems, patient health related information is measured by a set of wearable sensors. The measured value is then transmitted through a Master node to a local data base. The details of the main components used in the system are as follows:

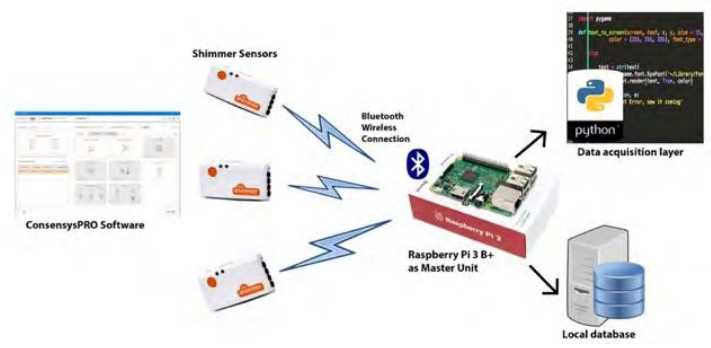

Figure 1. Remote health monitoring system architecture encompassing a Raspberry Pi and Shimmer sensors 
Shimmer devices (medical sensors). As previously stated, Shimmer is a wearable physiological sensing device used to monitor the status of various health parameters. Shimmer wireless sensing technology offers two modes for data acquisition. Measured data can be either stored locally on the sensing unit or streamed through Bluetooth to the Master node. The Shimmer3 device is equipped with a microcontroller, a built in microSD Flash socket for additional storage and Bluetooth v2.1 + Enhanced Data Rate (EDR) radio module [MICROCHIP, 2015]. In Bluetooth Classic, there are 79 channels, each with a channel bandwidth of $1 \mathrm{MHz}$ and a raw symbol rate of $1 \mathrm{M} \mathrm{symbol} / \mathrm{s}$. The modulation scheme could be Gaussian frequency shift keying (GFSK), quadrature phase shift keying (4PSK), or 8PSK [Chang, 2014]. The maximum sampling rate in the Shimmer3 device is $2048 \mathrm{~Hz}$ that can run up to 10 hours at a single battery charge [Gardašević et al., 2017].

Master node and the local database. The preliminary role of a Master node is to create a piconets topology with Shimmer3 sensing devices. It can also store streamed data of physiological devices locally. The Raspberry Pi B+ is the latest model of the Raspberry Pi series (released 2018) that were used as a Master node. The main reason for using Raspberry $\mathrm{Pi}$ is the low cost of employing this device at home environment for remote health monitoring. The optimal operating system for use in the Raspberry Pi is Raspbian ${ }^{1}$, which is based on Debian/Linux operating system. The free operating system "Raspbian stretch with desktop" comes with a graphical user interface (GUI) and a pre-installed version of the Python language. The most remarkable hardware update related to the Raspberry $\mathrm{Pi} \mathrm{B}+$ is its chipset for wireless connectivity that supports Bluetooth 4.2 Low Energy (BLE) [MagPi, 2018].

Consensys. Consensys is a free license software that is used to configure Shimmer devices. It is compatible with Microsoft operating system. There are several features that are provided by Consensys-Basic ${ }^{2}$ including, changing the sampling rate, managing the type of data collection and real-time data streaming.

Data acquisition layer. The streamed data by Shimmer3 devices is sent to an open source Python script. The part of the Python script that is responsible for creating communication with the Shimmer3 devices was developed by the Shimmers Research Group [Shimmer RG., 2018]. We developed the second part of the script that allows storing the data locally on the Master unit (Raspberry Pi in this setup). The Python script comes with some standard libraries that can be executed on many different operating systems such as Windows, macOS and most distributions of Linux.

\section{EVALUATIONS}

To evaluate the robustness of the designed architecture, four experiments were carried out by using different configurations of the control system. This section explains the experimental setup and the results from real world testbed.

Experimental setup. We conducted a set of experiments to evaluate the system performance in terms of packet received ratio (PRR) in different environments: (i) at home with low level of interference, and (ii) at work with higher level of interference. We selected university library to simulate the noisy working environment since more WiFi access points and larger number of users are generally exist. A spectrum analyzer was employed to discover the surrounding WiFi signals that could potentially create interference in our experiments.

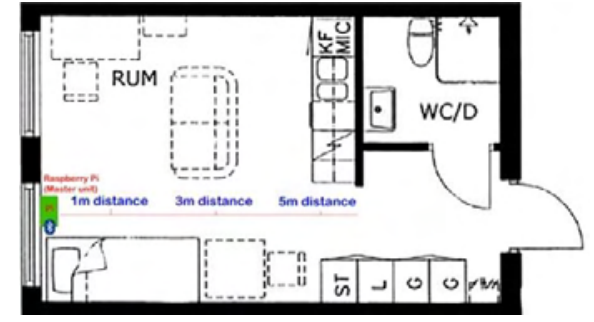

Figure 2. Position of the Master unit and the Shimmer devices from different distances

\footnotetext{
${ }^{1}$ https://www.raspberrypi.org/downloads/raspbian/

2 http://www.shimmersensing.com/products/consensys
} 
Figure 2 shows the positions of the Master node and the Shimmer devices at home environment. As it is shown, the Shimmer devices have been located in different proximity of the Master node, $1 \mathrm{~m}, 3 \mathrm{~m}$ and $5 \mathrm{~m}$. Besides the distance, we have also evaluated the network performance based on the number of shimmer devices in the piconet, sensors' sampling rates, and also the effect of human body presence and the mobility.

Impact of number of nodes. As each health-monitoring application may need a specific number of physiological sensors, varying the number of sensors in the experiment is one of the first steps towards system evaluation. We configured the Shimmer devices by using Consensys-Basic. The sampling rate was set to $512 \mathrm{~Hz}$. The 18-byte Bluetooth frame encompasses the data of low-noise accelerometer, wide-range accelerometer, and battery voltage. This frame size format is also appropriate for sending ECG, EMG or respiration measurements. We conducted experiments with different number of nodes, while nodes were locating in different distances from the Master node. Each experiment was run for a duration of 10 min. the stored data of sensing measurements and RSSI values were stored in files in the Master node.

Figure 3 to 8 shows the performance of the network in terms of PRR together with the relative RSSI value. The three numbers in the RSSI value graph represents the maximum, average and minimum reading of nodes' RSSI values for each scenario. The results from Figure 4 shows a high value of RSSI reading from all Shimmer devices in $1 \mathrm{~m}$ distance. The high PRR values (nearly 100\% packet deliver) reveals that there is a high correlation between the RSSI and PRR when the RSSI is strong. Thus, keeping the Master node closer to physiological devices regardless on number of nodes would result in a reliable system. It is also important to note that we considered $512 \mathrm{~Hz}$ sampling rate, which is for example, the minimum required frequency for collecting ECG parameter in order to monitor heart condition.

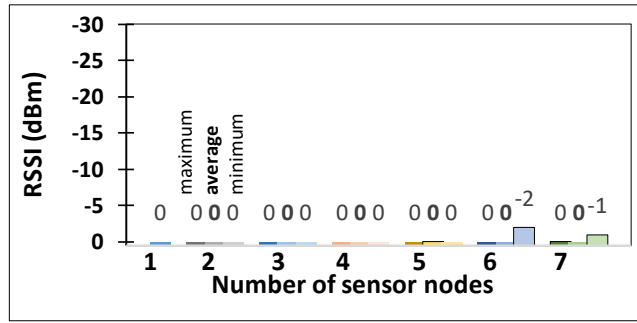

Figure 3. Measured RSSI values for different number of sensors located in $1 \mathrm{~m}$ distance from the Master node

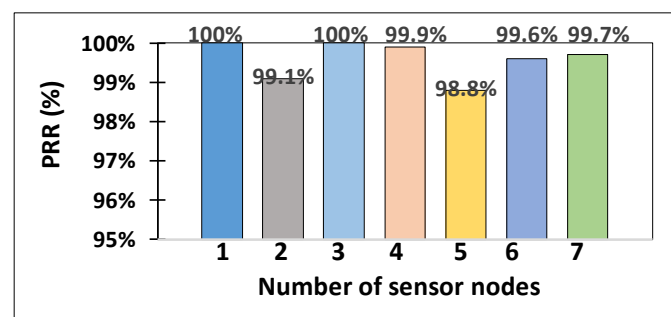

Figure 4. Packet reception rate for different number of sensors located in $1 \mathrm{~m}$ distance from the Master node

Figure 5 and 6 illustrate the results of RSSI and PRR when sensor nodes are located $3 \mathrm{~m}$ far from the destination. In this scenario we observe some fluctuation in the RSSI value as well as a sudden drop in signal strength. Moreover, the higher the number of nodes the lower the PRR, meaning that by increasing the number of physiological sensors there is a meaningful reduction in successful data transmission.

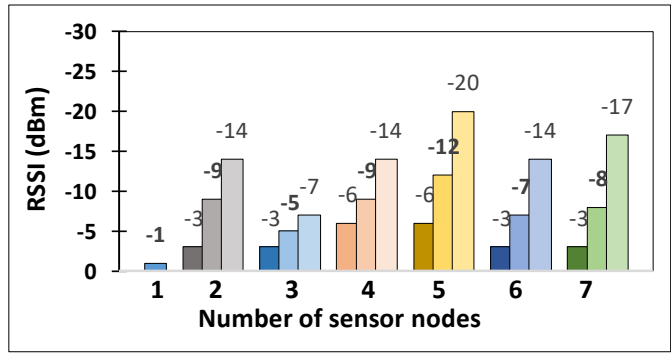

Figure 5. Measured RSSI values for different number of sensors located in $3 \mathrm{~m}$ distance from the Master node

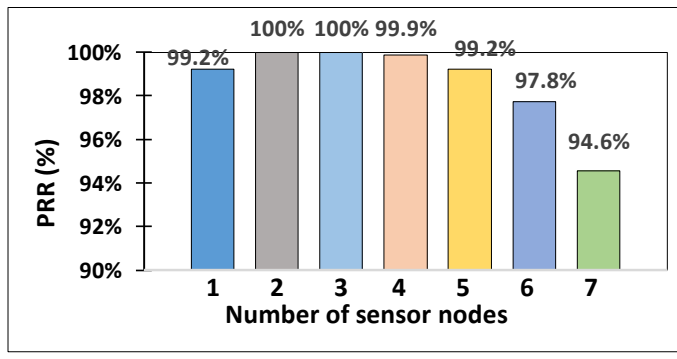

Figure 6. Packet reception rate for different number of sensors located in $3 \mathrm{~m}$ distance from the master node 
Further increase in the distance between sensor nodes and the Master node degrades system performance as illustrated in Figure 7 and 8 . However, the system is still able to provide high reliability with low number of nodes (less than 4 nodes). This justifies that the transmission power of the Bluetooth radio is strong enough to provide reliable links up to $5 \mathrm{~m}$ distance. We have limited our experiments up to $5 \mathrm{~m}$ distance as there were space limitation in the experiment area.

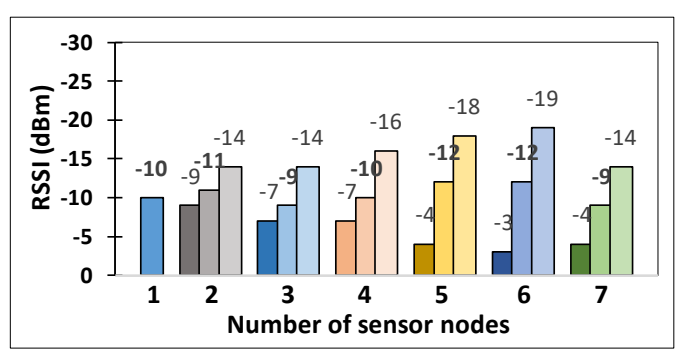

Figure 7. Measured RSSI values for different number of sensors located in $5 \mathrm{~m}$ distance from the Master node

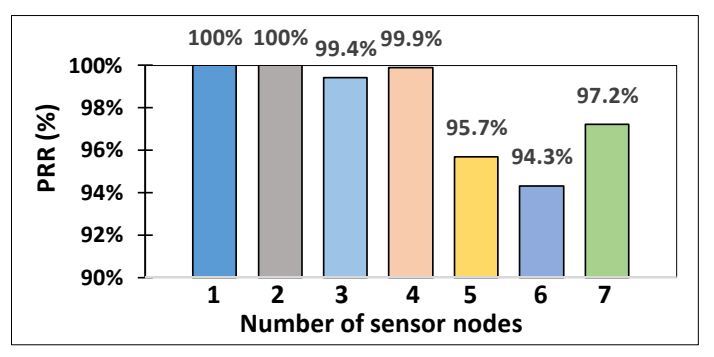

Figure 8. Packet reception rate for different number of sensors located in $5 \mathrm{~m}$ distance from the Master node

Impact of sampling rate. Sampling rate is one of the major parameters that could have high impact on the overall network performance. We run a set of experiment to evaluate the network performance with different sampling rate of $256 \mathrm{~Hz}, 512 \mathrm{~Hz}$ and $1024 \mathrm{~Hz}$. We used the maximum possible Shimmer devices for this set of experiments ( 7 Shimmers) while varying the distance from $1 \mathrm{~m}$ to $5 \mathrm{~m}$.

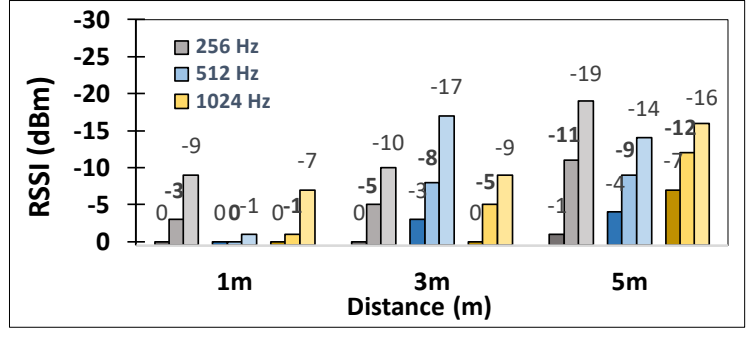

Figure 9. Measured RSSI values with different sampling rates of $256 \mathrm{~Hz}, 512 \mathrm{~Hz}$ and $1024 \mathrm{~Hz}$ when varying the distance to Master node from 1 to $5 \mathrm{~m}$

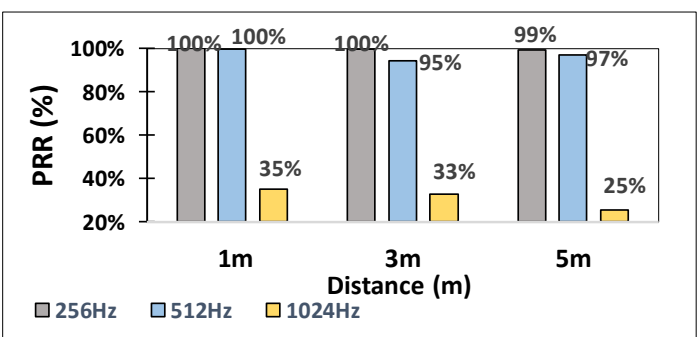

Figure 10. Packet reception rate with different sampling rates of $256 \mathrm{~Hz}, 512 \mathrm{~Hz}$ and $1024 \mathrm{~Hz}$ when varying the distance to Master node from 1 to $5 \mathrm{~m}$

Figure 9 shows that regardless of the chosen sampling rate, the RSSI has more fluctuations and lower strength in longer distances. However, as shown in Figure 10 there is a high correlation between the PRR and the sampling rate. As expected increasing the sampling rate to $1024 \mathrm{~Hz}$ would significantly degrade the network performance in all distances. This experiment further proves that sampling rate is one of the crucial parameters that must be tune properly in order to maintain a reliable system.

Impact of human body presence. Human body has been known as one of the major sources of interference for sensor devices. To investigate the impact of human body, we attached seven Shimmer devices on a person and ask the person to stand at the selected distances from the Master node during the experiment while wearing the sensors.

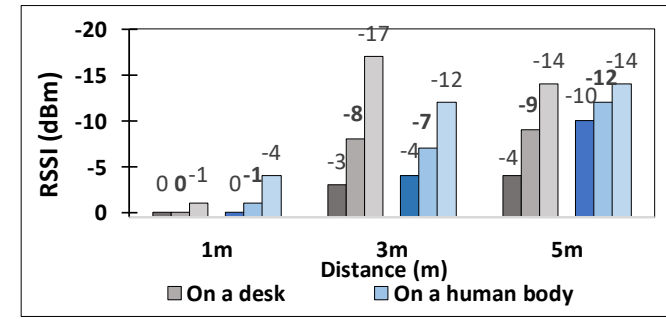

Figure 11. Measured RSSI with $512 \mathrm{~Hz}$ sampling rate when locating either on a desk or attached on human body

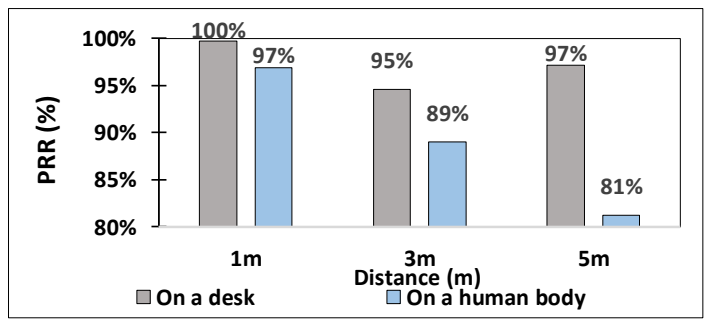

Figure 12. Packet reception rate with $512 \mathrm{~Hz}$ sampling rate when locating either on a desk or attached on human body 
Results from Figure 11 and 12 show the RSSI and PRR values for scenarios where Shimmer devices are either located on a desk or attached to a human body. Unlike our expectation, we see quite similar results for measured RSSI value in both scenarios, meaning that the human body has no significant impact on the RSSI value. Thanks to the strong transmission power that covers the weak interference caused by the human body. However, we observe a reduction in the network reliability by having less PRR when nodes are attached to human body. This observation makes it clear that although the RSSI value remained fairly unchanged but the higher level of noise floor caused by the presence of a human body would degrade the system performance when the RSSI value is relatively low (ie, at $5 \mathrm{~m}$ distance).

Impact of mobility. We evaluated the performance of the system in a more realistic scenario, where the Shimmer devices are worn by a person. We considered two cases: (i) the person with attached sensors is standing at a fixed position in a $5 \mathrm{~m}$ distance from the Master node, and (ii) the person walks randomly with a normal human walk speed in a room, up to $5 \mathrm{~m}$ distance from the Master node. We compare these two cases with the scenario where the Shimmer devices were located on a desk locating $5 \mathrm{~m}$ far from the Master node.

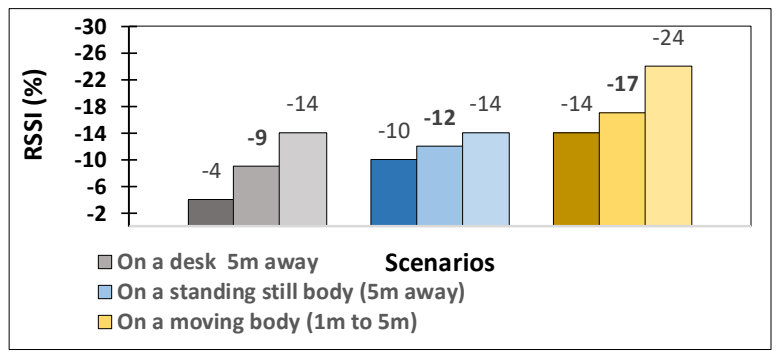

Figure 13. Measured RSSI values with $512 \mathrm{~Hz}$ sampling rate for the static and mobile scenarios

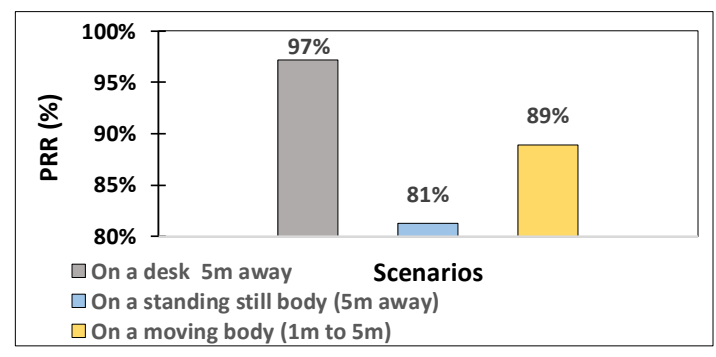

Figure 14. Packet reception rate with $512 \mathrm{~Hz}$ sampling rate for the static and mobile scenarios

Figure 13 once more shows that the RSSI drops gradually when sensors are attached on the body, but it degrades more when the person moves randomly in the room. The minimum RSSI value in the mobile scenario reaches to $-24 \mathrm{dBm}$, which is a meaningful drop comparing to its counterpart in the static scenario. The same pattern is seen as before in the PRR. Figure 14 reveals that the system reliability degrades when sensors are attached to the human body. However, we noticed more packet drops for the case where the person is standing at a fixed position. The reason is that we have selected the worst-case scenario, where the person is standing $5 \mathrm{~m}$ away from the Master node, while in a mobile scenario, the person is moving randomly in the room with the maximum length of $5 \mathrm{~m}$.

Impact of interference. As stated earlier, wireless networks that operate in $2.4 \mathrm{GHz}$ are the main sources of interference for the Bluetooth-enabled network. In this work, we conducted another set of experiments at a library environment at MDH university. We conducted the tests during a busy period of the day at the library, in order to provide similar noise condition as in a hospital. Besides people using their mobile phones, at the hospital, patients' medical information and images are transferred through WiFi access points.

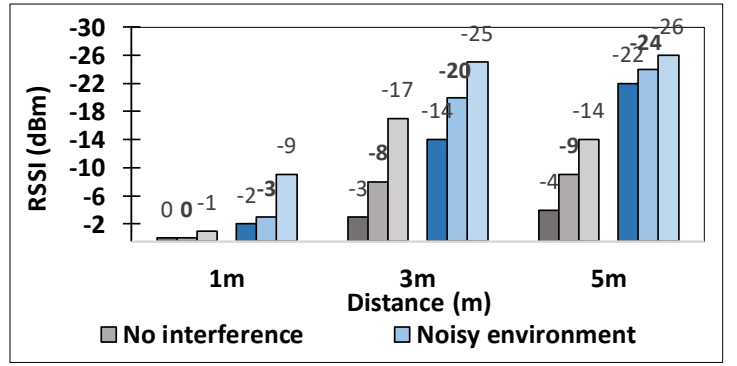

Figure 15. Measured RSSI in a non-noisy scenario with a noisy scenario at library in a static network when locating Shimmer devices in a 1 to $5 \mathrm{~m}$ distance

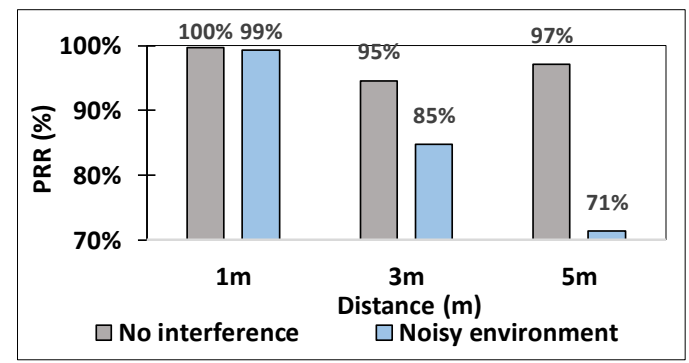

Figure 16. Packet reception rate in a non-noisy scenario with a noisy scenario at library in a static network when locating Shimmer devices in a 1 to $5 \mathrm{~m}$ distance 
Figure 15 shows that the RSSI value reduces remarkably in a library environment as the distance between the sensors and the Master node grows. RSSI reduction is mainly due to the presence of people and the furniture that attenuate the signal. Figure 16 shows that the system can be still reliable in a noisy environment if the Shimmer devices and the Master node are at most $1 \mathrm{~m}$ apart. Otherwise, up to $30 \%$ of data can be lost which is not acceptable in the critical health monitoring applications.

\section{CONCLUSIONS}

In this work, we have extensively evaluated the performance of Shimmer devices in terms of wireless connectivity. Shimmer devices are equipped with Bluetooth classic radio that operates in $2.4 \mathrm{GHz}$ frequency, sharing the same frequency band with WiFi. The main goal of the experiment was to study the impact of different sampling rate, presence of human body, mobility and interference on the overall system performance. We found that the Bluetooth radio is reliable at home environment where the distance between the sensor devices and the Master node does not exceed $5 \mathrm{~m}$. The maximum sampling rate to achieve high PRR is $512 \mathrm{~Hz}$, which is sufficient for EXG signal monitoring with streaming requirement. The presence of human body can degrade the system performance. Mobility of the person is another source of performance degradation, where we recorded about $9 \%$ more packet losses when a person with attached sensors moves randomly. Finally, the extreme noisy environment, such as in hospital and library, degrades the network performance drastically. We observed $10 \%$ more packet losses in $3 \mathrm{~m}$ distance and $25.8 \%$ more packet losses in $5 \mathrm{~m}$ distance.

\section{ACKNOWLEDGEMENT}

The work presented in this paper is supported by the Swedish Research Council (Vetenskapsrådet) through starting grant no. 2018-04582 via the MobiFog project, and by the Swedish Knowledge Foundation (KKS), through Prospekt grant via the FlexiHealth project and research profile ESS-H plus, and the EU Celtic_Plus/Vinnova project, Health5G (Future eHealth powered by 5G).

\section{REFERENCES}

Ahmed, M. U., Fotouhi, H., Köckemann, U., Lindén, M., Tomasic, I., Tsiftes, N., \& Voigt, T. (2017). Run-time assurance for the e-care@ home system. International Conference on IoT Technologies for HealthCare, 107-110. Springer.

Alam, M. M., Hamida, E. B., Rehmani, M. H., \& Pathan, A. S. K. (2016). Wearable wireless sensor networks: Applications, standards, and research trends. Emerging Communication Technologies Based on Wireless Sensor Networks: Current Research and Future Applications, 59-88. CRC Press.

Baker, N. (2005). ZigBee and bluetooth: Strengths and weaknesses for industrial applications. Computing and Control Engineering, 16(2), 20-25.

Chang, K. H. (2014). Bluetooth: A viable solution for IoT? [Industry Perspectives]. Wireless Communications, 21(6), 6-7. IEEE.

Chen, Y., \& Kunz, T. (2016). Performance evaluation of IoT protocols under a constrained wireless access network. International Conference on Selected Topics in Mobile \& Wireless Networking, 1-7. IEEE.

Doukas, C., \& Maglogiannis, I. (2012). Bringing IoT and cloud computing towards pervasive healthcare. International Conference on Innovative Mobile and Internet Services in Ubiquitous Computing, 922-926. IEEE.

Fotouhi, H., Vahabi, M., Ray, A., \& Björkman, M. (2016). Reliable communication in health monitoring applications. International Conference on IoT Technologies for HealthCare, 64-70. Springer.

Gardašević, G., Fotouhi, H., Tomasic, I., Vahabi, M., Björkman, M., \& Lindén, M. (2017). A heterogeneous IoT-based architecture for remote monitoring of physiological and environmental parameters. International Conference on IoT Technologies for HealthCare, 48-53. Springer.

MagPi. (2018). Raspberry PI 3B+ specs and benchmarks. Retrieved May 2019 from https://www.raspberrypi.org/magpi/raspberry-pi-specs-benchmarks/. 
MICROCHIP. (2015). RN42/RN42N, class 2 Bluetooth ${ }^{\circledR}$ module with EDR support. Retrieved May 2019 from http://ww1.microchip.com/downloads/en/DeviceDoc/50002328A.pdf.

Ministry of Health and Social Affairs. (2016). Vision for eHealth 2025. Retrieved May 2019 from https://www.government.se/information-material/2016/08/vision-for-ehealth-2025/.

Natarajan, R., Zand, P., \& Nabi, M. (2016, October). Analysis of coexistence between IEEE 802.15. 4, BLE and IEEE 802.11 in the $2.4 \mathrm{GHz}$ ISM band. International Conference of the IEEE Industrial Electronics Society, 6025-6032.

Premsankar, G., Di Francesco, M., \& Taleb, T. (2018). Edge computing for the internet of things: A case study. IEEE Internet of Things Journal, 5(2), 1275-1284.

Ray, P. P. (2018). A survey on internet of things architectures. Journal of King Saud University-Computer and Information Sciences, 30(3), 291-319.

Reiter, G. (2014). Wireless connectivity for the internet of things. Europe, 433, 868MHz.

Santos, J., Rodrigues, J. J., Silva, B. M., Casal, J., Saleem, K., \& Denisov, V. (2016). An IoT-based mobile gateway for intelligent personal assistants on mobile health environments. Journal of Network \& Computer Applications, 71, 194-204.

Sarkar, S., \& Misra, S. (2016). From micro to nano: The evolution of wireless sensor-based health care. IEEE pulse, 7(1), 21-25.

Shimmer, RG. (2018) shimmer3/LogAndStream/python_scripts/. Retrieved May 2019 from https://github.com/ShimmerResearch/shimmer3.

Shimmer. (2019). Consensys GSR Development Kits. Retrieved May 2019 from http://www.shimmersensing.com/products/gsr-optical-pulse-development-kit.

Tomasic, I., Petrović, N., Fotouhi, H., Lindén, M., \& Björkman, M. (2017). Data flow and collection for remote patients monitoring: from wireless sensors through a relational database to a web interface in real time. European Medical and Biological Engineering Conference (EMBEC) and the Nordic-Baltic, 89-92. Springer.

United Nations. (2013). World population ageing. Department of Economic and Social Affairs, Population Division. 